\title{
Effects of the Supercritical Fluid Extraction of Dahurian Angelica Root and Szechwan Lovage Rhizome on Spontaneous Hypertension Rats ${ }^{*}$
}

\author{
Yan Zhang ${ }^{1 \#}$, Fenxia Gao ${ }^{1}$, Yanjun $\mathrm{Cao}^{2}$, Hongying Wang ${ }^{2}$, Haijie Duan ${ }^{3}$ \\ ${ }^{1}$ The Mental Health Center of Xi'an, Xi'an, China \\ ${ }^{2}$ School of Medicine, Xi'an Jiaotong University, Xi'an, China \\ ${ }^{3}$ No. 5 Hospital of Xi'an, Xi'an, China \\ Email: "classicyan@gmail.com
}

Received October 15, 2012; revised November 16, 2012; accepted November 24, 2012

\begin{abstract}
The supercritical fluid extraction of Dahurian Angelica Root (Bai Zhi) and Szechwan Lovage Rhizome (Chuan Xiong) was named as BCC. In the study, we investigated whether BCC had effects on left ventricular hypertrophy (LVH) and myocardial fibrosis in spontaneous hypertensive rats (SHR). For SHR + BCC group, BCC $(0.3 \mathrm{~g} / \mathrm{kg})$ was orally administered daily for 12 weeks. The SHR group and the Wistar Kyoto rats (WKY, normal control) group, the equal volume of 5\% CMC-Na distilled water. After 12 weeks, left ventricle was segregated from each rat in the groups, and the left ventricle weight/body weight (LVW/BW) calculated. The volume fraction of collagen (VFC) in myocardium and the diameter of cardiac muscle cell (DCMC) were examined by histological staining. Biochemical indicators of blood sample such as Angiotensin II (Ang II), Aldosterone (ALD), Hyaluronic Acid (HA), Laminin (LN), Procollagen III (PC III) and Collagen type IV (CIV) levels were detected by using radioimmunoassay (RIA). And also NOS and iNOS levels were measured by means of ultra-violet spectroscopy (UV). The results shown that in SHR + BCC group, the LVW/BW, DCMC and VFC decreased significantly versus SHR group, the same as biochemical indicators except NOS and iNOS. All of above index was similar to WKY group. Statistically significant correlations were found among the plasma Ang II level, the mean systolic blood pressure (SBP), and the NOS level of the three groups. Our study indicates that the BCC can control the LVH and myocardial fibrosis in SHR.
\end{abstract}

Keywords: Dahurian Angelica Root; Szechwan Lovage Rhizome; LVH; Myocardial Fibrosis; SHR

\section{Introduction}

Hypertension is one of the most significant risk factors in the development and progression of a variety of cardiovascular diseases including cardiac hypertrophy and myocardial fibrosis. Clinical studies suggest that a high arterial pressure is associated with myocardial hypertrophy and interstitial fibrosis [1]. In the hypertensive state, a number of adaptive changes occur in heart [2] such as left ventricular hypertrophy (LVH) and myocardial interstitial fibrosis. At the same time, these structural abnormalities may play an important role in the development and maintenance of hypertension [3,4]. In addition, some studies shown that the Angiotensin II (Ang II), Aldosterone (ALD) Hyaluronic Acid (HA), Laminin (LN), Procollagen III (PC III) and Collagen type IV (CIV) levels had an increase in myocardial interstitial fibrosis [5].

"Effects of the supercritical fluid extraction of Dahurian Angelica Root and Szechwan Lovage Rhizome on spontaneous hypertension rats (dispensable).

${ }^{\#}$ Corresponding author.
The effectors/hormones of Ang II and ALD could stimulate fibroblast-mediated collagen synthesis [6,7], and Ang II additionally suppresses collagenase activity [6], which synergistically leads to myocardial collagen accumulation, induces HA, LN, PCIII, CIV increased. Recent study showed reduced NOS derived NO production contributes to the hypertrophic growth and phenoltype of cardiac muscle cells [8]. All of these changes would aggravate $\mathrm{LVH}$, and exacerbate hypertension.

In clinic, some drugs such as angiotensin-converting enzyme inhibitors, angiotensin II receptor antagonists and calcium channel antagonists etc can not only decrease blood pressure, but also reverse the LVH and myocardial interstitial fibrosis. In recently years, some traditional Chinese medicines were reported to be effective against the hypertension [9]. Previous studies in our lab indicated that the Bai-Chuan capsule (BCC), a complex prescription of supercritical fluid extraction of Dahurian Angelica Root (Bai Zhi) and Szechwan Lovage Rhizome (Chuan Xiong) as a traditional Chinese medi- 
cine, in different dosages $(0.6,0.3$ and $0.15 \mathrm{~g} / \mathrm{kg})$ could obviously reduce the blood pressures in SHR $[10,11]$. In this paper, we will investigate the effects of the BCC on left ventricular hypertrophy (LVH) and cardiac fibrosis as an anti-hypertensive medicine in SHR. Therefore, we treated 14-week-old male SHR with chronic hypertension, advanced $\mathrm{LVH}$, and myocardial fibrosis, as well as age- and sex-matched normotensive WKY for 3 months with BCC. It was the first report about the effects of $\mathrm{BCC}$ on the structure of $\mathrm{LVH}$ and myocardial fibrosis in SHR.

\section{Materials and Methods}

\subsection{Composition of BCC}

BCC was prepared with the supercritical fluid extraction of Dahurian Angelica Root (Bai Zhi) and Szechwan Lovage Rhizome (Chuan Xiong) according to 1:1, which was provided by our lab and named as Bai-Chuan Capsule (BCC). Briefly, BCC improved in the Chinese folk medicine "Du Liang Wa", which had been used for over 1000 years in China. Prescriptions can be found in "Shi Zhai Bai Yi Xuan Fang" by Miao Wang (during the Song Dynasty) and recent drug standards issued by Beijing in 1983.

\subsection{Protocol}

Sixteen male SHR, age of 14 weeks, $270-290 \mathrm{~g}$, and eight male WKY rats, age of 14 weeks, 280 - $320 \mathrm{~g}$ were purchased from Chengdu Da Shuo Biological Technology Co., Ltd. (Chengdu, China). Rats were housed in temperature $\left(24^{\circ} \mathrm{C} \pm 2{ }^{\circ} \mathrm{C}\right)$ and humidity $(50 \% \pm 10 \%)$ controlled room with a $12-\mathrm{h}$ on/12-h off light cycle. Solid rodent chow and autoclaved water were given ad libitum.

Animals were allowed a period of 1 week of acclimatization prior to entry into any experimental protocol. To obtain an accurate blood pressure reading, rats remained still and unperturbed throughout the measurement period. Rats were conditioned to the restraint and the warming chamber for $10-20 \mathrm{~min} /$ day of $37^{\circ} \mathrm{C}$ before measurements. After 1 week, 16 SHR were randomly divided into two groups: SHR group and SHR + BCC group $(\mathrm{n}=$ 8 equally). Eight WKY rats were the normal control group. Rats in the SHR + BCC group were intragastric administration $\mathrm{BCC}$ at $0.3 \mathrm{~g} / \mathrm{kg}$ (equal to 11 -fold of clinic dosage), which was suspended in $5 \mathrm{ml}$ of 5\% CMC-Na distilled water, once a day for 12 weeks. The WKY and the SHR control groups were administrated an equal volume of 5\% CMC-Na distilled water.

\subsection{Measuring of Systolic Blood Pressure (SBP)}

The SBP was measured once a week for the first 3 weeks, and then measured fortnightly till the end of the experiment (eight times totally). After training period, SBP was measured in the morning after intragastric administration 2 hour. The maximum pressure of inflation was set 20 $40 \mathrm{mmHg}$ above anticipated SBP. The instrument was set for a maximum inflation pressure of $160 \mathrm{mmHg}$ for WKY rats that were expected to have pressures in the normal range and was increased to 250 or $300 \mathrm{mmHg}$ for markedly SHR rats. A typical run involved 6 repetitions of the automated inflation-deflation cycle with a 2-minute interval till Rats' pulsatory signals from the arteria caudilis were displayed steadily, then the mean of 3 readings within a $5-10 \mathrm{mmHg}$ range was taken as SBP of rat.

\subsection{Measurement of Left Ventricular Hypertrophy Ratio}

Twelve weeks later, the rats were weighed and anesthetized with $20 \%$ Ethylurethanm $(5 \mathrm{ml} / \mathrm{kg}$, intraperitoneal injection). The chest cavity was rapidly opened, and the heart was removed and rinsed in two washes of ice-cold saline. Major blood vessels and connective tissue was removed, the heart blotted dry, weighed, and the LVW/ BW ratio calculated. Other organ weights were determined and weight to total body weight ratios calculated.

\subsection{Myocardial Histological and Morphological Assay}

Part of the left ventricle, about $0.5 \mathrm{~cm}$ above apex cordis, was segregated, fixed in $4 \%$ paraformaldehyde, embedded in paraffin, sectioned at $4 \mu \mathrm{m}$, and stained with Hematoxylin-Eosin (HE) and Weigert Hematoxylin-Victoria blue'B-Ponceau. Myocardial slices, 16 of those round or similar to round were chose randomly from each group. The morphometric result for each section was the average of measurements from its volume fraction of collagen (VFC) and the diameter of cardiac muscle cell (DCMC). Because of the lack of universal consensus on the definition of myocardium VFS, animals with a $>30 \mu \mathrm{m}$ width collagen were classified as $2 /$ severe myocardial fibrosis, $5-30 \mu \mathrm{m}$ as $1 /$ medium, and $<5 \mu \mathrm{m}$ as $0 /$ normal myocardium. At the same time, Myocardial hypertrophy was defined DCMC $>45 \mu \mathrm{m}$ as $2 /$ severe hypertrophy, $30-45 \mu \mathrm{m}$ as $1 /$ medium, and $<30 \mu \mathrm{m}$ as $0 /$ normal cardiac muscle cell.

\subsection{Measurement of Plasma Ang II, ALD, NOS and iNOS}

Blood samples from all of the subjects were obtained from abdominal aorta in the morning after overnight fasting and were collected in vacuum tubes with EDTA as an anticoagulant. After being centrifuged at $3000 \mathrm{rpm}$ for 20 minutes at $4^{\circ} \mathrm{C}$ immediately after collection, the plasma samples were then kept frozen at $-40^{\circ} \mathrm{C}$ until analyzed. Blood Ang II and ALD levels were measured 
by radioimmunoassay (RIA) with commercially available kits (Beijing North Institute of Biotechnique). NOS and iNOS levels were measured by ultraviolet spectrophotometry (UV) with NOS and iNOS assay kits (Nanjing Jiancheng Bioengineering Institute).

\subsection{Measurement of Serum HA, LN, PCIII and CIV}

Blood samples from all of the subjects were obtained from abdominal aorta in the morning after overnight fasting and were collected in vacuum tubes with nothing as an anticoagulant. Sera were centrifuged after standing for 4 hours at room temperature, and preserved at $-20^{\circ} \mathrm{C}$ before assays. The serum level of HA, LN, PCIII and CIV were detected by radioimmunoassay (RIA) with commercially available kits (Beijing North biology technique institute).

\subsection{Statistical Analysis}

Results are expressed as the mean \pm S.D. Student's t-test was used for comparison between groups. Values of $\mathrm{P}<$ 0.05 were considered statistically significant.

\section{Results}

\subsection{Comparison of LVW/BW, Left Ventricle Morphology and Histology among Groups}

At the end of the treatment, it was conspicuous in our study that LVW/BW of the untreated SHR was higher than WKY rats $(2.78 \pm 0.49 \mathrm{mg} / \mathrm{g}$ vs $1.93 \pm 0.71 \mathrm{mg} / \mathrm{g})$, and animals treated by BCC had significantly lower LVW/BW compared with untreated animals $(2.78 \pm 0.49$ $\mathrm{mg} / \mathrm{g}$ vs $3.35 \pm 0.83 \mathrm{mg} / \mathrm{g}, \mathrm{P}<0.01)$. VFC and DCMC of untreated SHR were significantly higher than of WKY rats, but decreased in the SHR + BCC group (Figure 1).

Weigert Hematoxylin-Victoria blue'B-Ponceau staining showed that the VFC and numeral density of SHR was more increasing; collagen fibers in media were hyperplasic, cardiac muscle cell was hypertrophy and round (Figures 2(A) and (D)). In the BCC group (Figures 2(B) and (E)), VFC was medium, the DCMC of cardiac muscle cell was smaller than untreated SHR, and shape was approximation to WKY rats (Figures $2(\mathbf{C})$ and $(\mathbf{F})$ ).

\subsection{Serum HA, LN, PCIII and CIV Levels}

Treatment with BCC caused significantly reduction in all the level of HA, LN and CIV. The recorded levels of serum HA, LN, PCIII and CIV in WKY and SHR are shown in (Figure 3). HA, LN, PCIII and CIV levels in the sera from SHR with fibrotic changes were significantly higher when compared to the WKY rats and treated SHR.

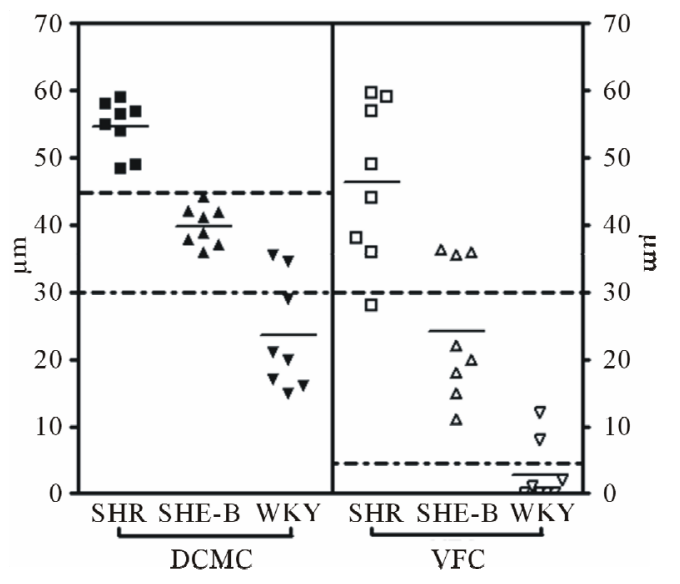

Figure 1. Photomicrographs demonstrating VFC, DCMC of SHR (SHR treated with BCC and WKY rats 12 weeks after surgery (G). 2 means severe myocardial fibrosis/severe cell hypertrophy, 1 medium, 0 normal.).

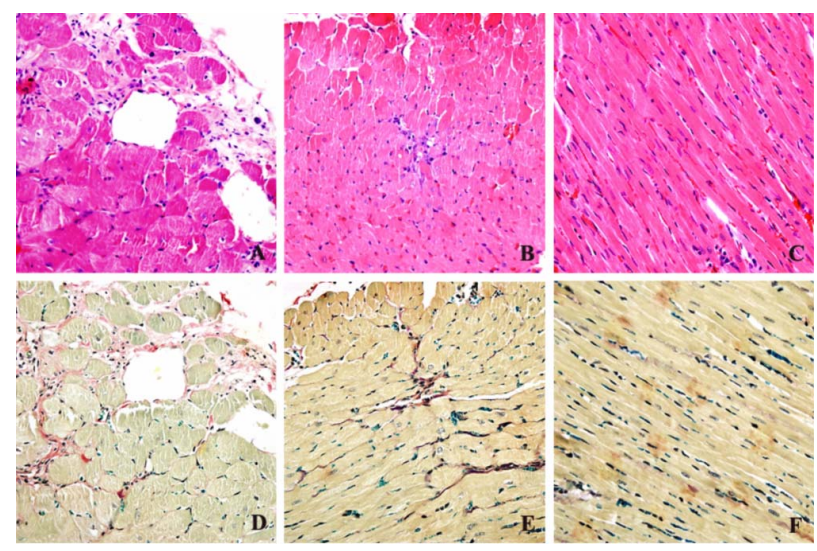

Figure 2. Heart sections of SHR staining (counterstained with HE staining (A, B and C) and Weigert Hematoxylin-Victoria blue'B-Ponceau staining (D, E, F). Magnification $\times 400$.).

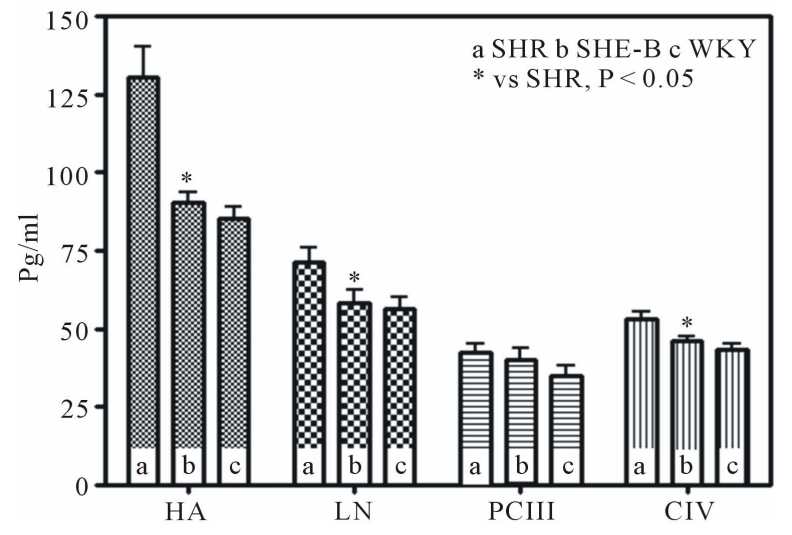

Figure 3. Serum HA, LN, PCIII and CIV levels (The recorded levels of serum HA LN, PCIII and CIV in Untreated SHR, treated SHR and WKY. There was difference in HA, LN, CIV between Untreated SHR and treated SHR $(\mathrm{P}<0.05)$.). 


\subsection{Blood Plasma Ang II, ALD, NOS and iNOS Levels}

Treatment with $\mathrm{BCC}$ caused significantly reduction in both the level of Ang II and ALD (Figure 4(a)), but augmentation in both the level NOS and iNOS of SHR. The untreated SHR, treated SHR and WKY showed inverse proportion for Ang II and NOS (Figure 4(c)). The proportion of iNOS and NOS in treated SHR were distinctive 1:2, but the iNOS level was about $90 \%$ of NOS in WKY (Figure 4(b)). Comparison of the three groups showed that untreated SHR had ALD of $298.3 \pm 57.7$ $\mathrm{pg} / \mathrm{ml}$, whereas the SHR treated with BCC demonstrated reduced ALD of $138.96 \pm 25.2 \mathrm{pg} / \mathrm{ml}$, which was near to WKY ALD of $100.18 \pm 38.8 \mathrm{pg} / \mathrm{ml}$ (Figure 4(a)).

\section{Discussion}

The results of our study showed an anti-hypertensive and a reversal of the heart remodeling effect of the supercritical fluid extraction of Dahurian Angelica Root and Szechwan Lovage Rhizome on SHR. The SHR treated with a daily dose of BCC over a period of 12 weeks not only demonstrated arrest of the development of hypertension, but a significant reduction myocardial fibrosis and coherent index. Cardiac hypertrophy is a well-established major risk factor for cardiovascular disease, including sudden death. But, we also found that that LVW/ BW of the untreated SHR was higher than WKY rats, and animals treated with BCC had significantly lower LVW/BW compared with untreated animals in the model group. Most antihypertensive drugs lower BP and also cause regression of LVH (Jennings et al., 1997). However, the classic arterial vasodilators, hydralazine and minoxidil, cause minimal regression [12] and can increase LV mass in hypertensive rats and humans. BCC not only can protect BP, but also can cause regression of LVH. Morphological studies indicate that the increment in myocardial mass is caused by muscle hypertrophy and a disproportionate accumulation of fibrillar collagen in the interstitial space. Aldosterone emerges as important determinants of myocardial fibrosis [13,14]. This factor increases the synthesis of collagen I and III by cardiac fibroblasts [6]. The direct pro-fibrotic effect of ALD is mediated through specific corticoid receptors in cardiac fibroblast [15], and is independent of cardiac load and LVH. In addition, experimental evidence has been collected on a cross-talk between ALD and ET. ET-1 has been demonstrated to stimulate ALD secretion, both in animals and in humans, having a direct secretagogue effect on the adrenal cortex [16], equipotent to that of Ang II, and ALD infusion in experimental models of salt-loaded rats has been shown to enhance ET-1 production [17]. The possible effect of ALD on myocardial performance is not obvious. Increased collagen content

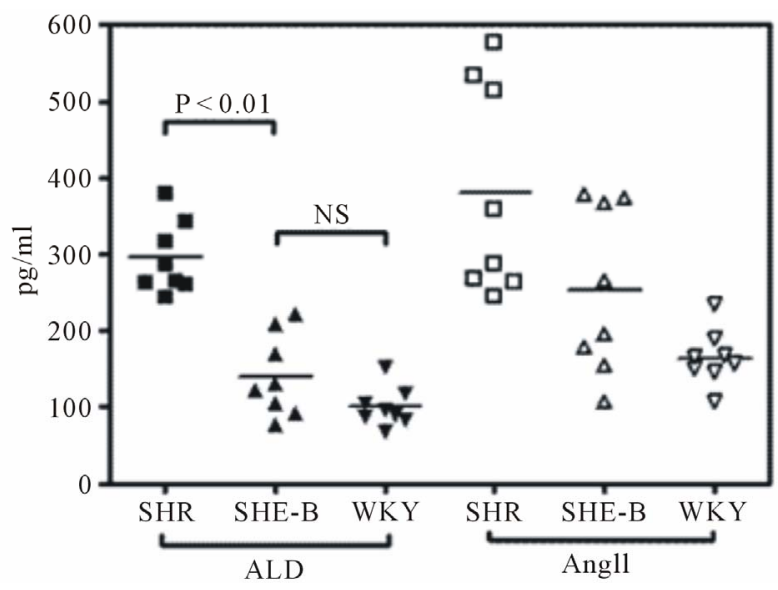

(a)

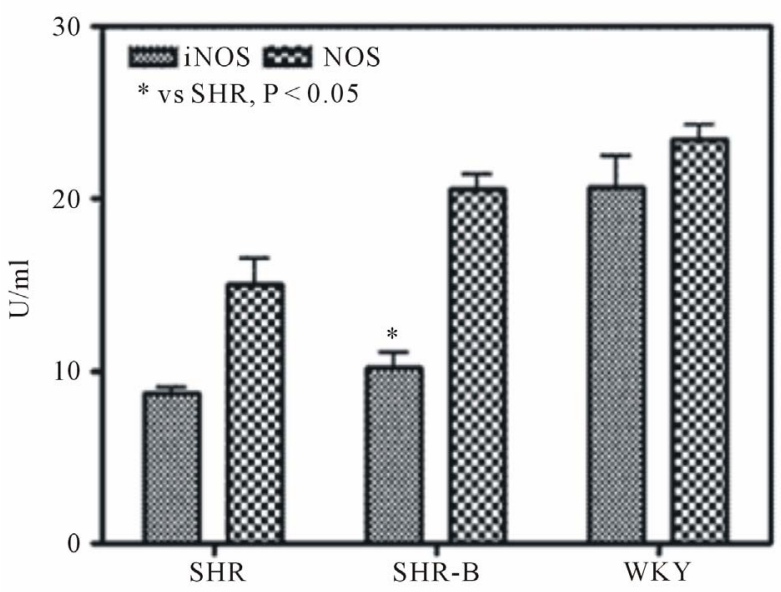

(b)

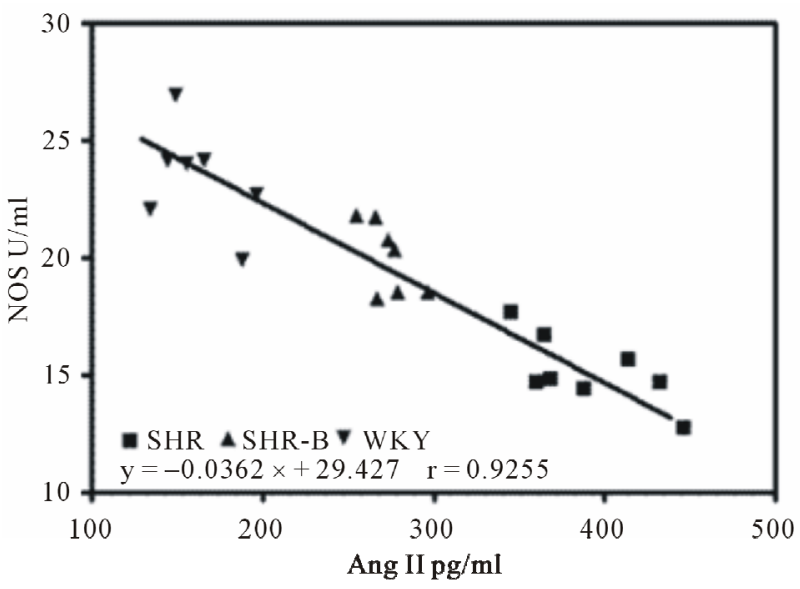

(c)

Figure 4. Blood plasma Ang II, ALD, NOS and iNOS levels (Comparison of Ang II and ALD in the three experimental groups (a). Comparison of NOS and iNOS in the three experimental groups (b). The correlation between NOS and plasma Ang II in Untreated SHR, treated SHR and WKY (c; $r=0.93 ; P<0.01)$. Data are expressed as the mean \pm S.D. (n $=8$ rats/group). ${ }^{*} \mathrm{P}<0.01$ means significantly different from SHR group.). 
within myocardial interstitium can be presumed to impair contractile behavior of myocardial fibers. Our data suggest that ALD-induced alterations in myocardial composition and geometry result in a subtle impairment of myocardial performance. These alterations can also modify myocardial stiffness and therefore LV diastolic properties. The left ventricle morphology and histology results show that collagen fibers in media were hyperplastic, elastic fibers decreased and disordered, part of which were substituted by collagen fibers.

Recently, study has demonstrated that macrophage infiltration is an early key event for reactive myocardial fibrosis, especially perivascular fibrosis in this model [18]. Also, it has been shown that Ang II supports leukocyte transmigration via AT1 receptor-dependent, but arterial pressure-independent, mechanisms [19,20]. Taken together, the present study provides in vivo evidence that Ang II might play an important role in early fibrotic changes and the resultant reactive myocardial fibrosis in hearts by activating the macrophage-mediated inflammatory process. Of course, Ang II is not the only proinflammatory mediator. Recent studies have suggested that it is a strong proinflammatory factors, because mechanical strain can induce inflammatory cytokines, growth factors, and oxidative stress, as well as tissue RAS in the vessel wall [21]. The interplay of these factors might regulate tissue Ang II production and the fibrotic process in hearts.

Our study clearly demonstrated that BCC supplementation could block the effects of high blood pressure on plasma Ang II, NOS level, Biochemical indicators of myocardial fibrosis levels, and BP in SHR. These observations indicated that BCC might influence NOS by inhibiting the Ang II production and preventing the BP elevation. The pharmacological results demonstrated that the effective component of BCC in CMC system can act on rat artery cell membrane as Verapamil, a calcium antagonist. And further studies showed that the effective component had the effects of inhibiting vasoconstriction in vitro on rat abdominal aorta segments. It would be our next research project.

\section{REFERENCES}

[1] K. Michaela, B. Simona, P. Carlo, B. Giampaolo, M. Angelica, F. Stefania, et al., "Myocardial Ultrasonic Backscatter in Hypertension Relation to Aldosterone and Endothelin," Hypertension, Vol. 41, No. 2, 2003, pp. 230236. doi:10.1161/01.HYP.0000052542.68896.2B

[2] Y. Hu and N. S. Cai, "Reconstitution of LVH in High Blood Pressure," Chinese Journal of Internal Medicine, Vol. 36, No. 6, 1997, pp. 424-426.

[3] L. M. De, A. Estevez, D. Bunout, C. Klenner, M. Oyonarte and S. Hirsch, "Ventricular Mass in Hypertensive and Normotensive Obese Subjects," International Jour- nal of Obesity and Related Metabolic Disorders, Vol. 18, No. 4, 1994, pp. 193-197.

[4] M. S. Lauer, K. M. Anderson and D. Levy, "Separate and Joint Influences of Obesity and Mild Hypertension on Left Ventricular Mass and Geometry: The Framingham Heart Study," Journal of the American College of Cardiology, Vol. 19, No. 1, 1992, pp. 130-134. doi:10.1016/0735-1097(92)90063-S

[5] C. Li and F. Lu, "Change of Myocardial Interstitial Fibrosis Index in Hypertension Complicated with Left Ventricular Hypertrophy and Influence of ACET Therapy," Chinese Journal of Cardiovascular Rehabilitation Medicine, Vol. 12, No. 1, 2003, pp. 22-24.

[6] C. G. Brilla, G. Zhou, L. Matsubara and K. T. Weber, "Collagen Metabolism in Cultured Adult Rat Cardiac Fibroblasts: Response to Angiotensin II and Aldosterone," Journal of Molecular and Cellular Cardiology, Vol. 26, No. 7, 1994, pp. 809-820. doi:10.1006/jmcc.1994.1098

[7] F. J. Villarreal, N. N. Kim, G. D. Ungab, M. P. Printz and W. H. Dillmann, "Identification of Functional Angiotensin II Receptors on Rat Cardiac Fibroblasts," Circulation, Vol. 88, No. 6, 1993, pp. 2849-2861.

doi:10.1161/01.CIR.88.6.2849

[8] W. Sibylle, R. Cornelia, W. Sandra, R. Joachim, K. Georg and S. Klaus-Dieter, "Lack of Endothelial Nitric Oxide Synthase-Derived Nitric Oxide Formation Favors Hypertrophy in Adult Ventricular Cardiomyocytes," $\mathrm{Hy}$ pertension, Vol. 49, No. 1, 2007, pp. 193-200.

[9] H. C. Shih, T. H. Lee, S. C. Chen, C. Y. Li and T. Shibuya, "Antihypertension Effects of Traditional Chinese Medicine Ju-Ling-Tang on Renal Hypertensive Rats," The American Journal of Chinese Medicine, Vol. 33, No. 6, 2005, pp. 913-921. doi:10.1142/S0192415X05003545

[10] W. H. Zhao, Y. X. Cao, J. Liu and L. C. He, "The Influence of Bai Chuan Capsule to Awake Rats," Journal of Xi' an Medical University, Vol. 22, No. 4, 2001, pp. 315336.

[11] Y. Zhang, L. C. He, H. J. Duan and Y. Z. Zhan, "Effects of Bai Chuan Capsule on Left Ventricular Hypertrophy and Correlative Indexes," Journal of Chinese Medicinal Materials, Vol. 33, No. 8, 2010, pp. 1290-1292.

[12] J. M. Cruickshank, J. Lewis, V. Moore and C. Dodd, "Reversibility of Left Ventricular Hypertrophy by Differing Types of Antihypertensive Therapy," Journal of Human Hypertension, Vol. 6, No. 2, 1992, pp. 85-90.

[13] N. Varo, M. J. Iraburu, M. Varela, B. Lùpez, J. C. Etayo and J. Dìez, "Chronic AT1 Blockade Stimulates Extracellular Collagen Type I Degradation and Reverses Myocardial Fibrosis in Spontaneously Hypertensive Rats," Hypertension, Vol. 35, No. 6, 2000, pp. 1197-1202. doi:10.1161/01.HYP.35.6.1197

[14] K. T. Weber and G. G. Brilla, "Pathological Hypertrophy and Cardiac Interstitium: Fibrosis and Renin-AngiotensinAldosterone System," Circulation, Vol. 83, No. 6, 1991, pp. 1849-1865. doi:10.1161/01.CIR.83.6.1849

[15] M. Lombès, N. Alfaidy, E. Eugene, A. Lessana, N. Farman and J. P. Bonvalet, "Prerequisite for Cardiac Aldosterone Action: Mineralcorticoid Receptor and 11-Hydroxysteroid Dehydrogenase in the Human Heart," Circula- 
tion, Vol. 92, No. 2, 1995, pp. 175-182.

doi:10.1161/01.CIR.92.2.175

[16] G. P. Rossi, A. Sacchetto, M. Cesari and A. C. Pessina, "Interaction between Endothelin-1 and Renin-Angiotensin-Aldosterone System," Cardiovascular Research, Vol. 43, No. 2, 1999, pp. 300-307.

doi:10.1016/S0008-6363(99)00110-8

[17] E. L. Schiffrin, "Role of Endothelin-1 in Hypertension and Vascular Disease," American Journal of Hypertension, Vol. 14, No. 6, 2001, pp. 83S-89S. doi:10.1016/S0895-7061(01)02074-X

[18] F. Kuwahara, H. Kai, K. Tokuda, H. Niiyama, N. Tahara, K. Kusaba, et al., "Roles of Intercellular Adhesion Molecule-1 in Hypertensive Cardiac Remodeling," Hypertension, Vol. 41, No. 3, 2003, pp. 819-823.

[19] W. B. Strawn, P. E. Gallagher, E. A. Tallant, D. Ganten and C. M. Ferrario, "Angiotensin II AT1-Receptor Blockade Inhibits Monocyte Activation and Adhesion in Transgenic (mRen2) 27 Rats," Journal of Cardiovascular Pharmacology, Vol. 33, No. 3, 1999, pp. 341-351. doi:10.1097/00005344-199903000-00001

[20] L. Pastore, A. Tessitore, S. Martinotti, E. Toniato, E. Alesse, M. C. Bravi, et al., "Angiotensin II Stimulates Intracellular Adhesion Molecule-1 (ICAM-1) Expression by Human Vascular Endothelial Cells and Increases Soluble ICAM-1 Release in Vivo," Circulation, Vol. 100, No. 15, 1999, pp. 1646-1652. doi:10.1161/01.CIR.100.15.1646

[21] A. Nicoletti and J. B. Michel, "Cardiac Fibrosis and Inflammation: Interaction with Hemodynamic and Hormonal Factors," Cardiovascular Research, Vol. 41, No. 3, 1999, pp. 532-543. doi:10.1016/S0008-6363(98)00305-8 